\title{
Management of Recurrent Optic Disc Pit-Associated Maculopathy with Human Amniotic Membrane Graft in a Pediatric Case: A Case Report
}

\author{
Aditya Kelkar Jai Aditya Kelkar Mounika Bolisetty Ashwani Kanoriya \\ Department of Ophthalmology, National Institute of Ophthalmology, Pune, India
}

\section{Keywords}

Human amniotic membrane graft · Intraoperative ocular coherence tomography · Optic disc pit - Maculopathy $\cdot$ Recurrence $\cdot$ Pediatric case

\begin{abstract}
A 13 years old previously operated with $25-\mathrm{G}$ pars plana vitrectomy with double internal limiting membrane peel and juxtapapillary endolaser with SF 6 gas tamponade for optic disc pitassociated maculopathy (ODP-M), presented with recurrence after 9 months of successful primary surgery. Three 25-G sclerotomies were made and Human Amniotic Membrane Graft was tucked into the optic disc pit (ODP), the position of the graft was confirmed with intraoperative ocular coherence tomography. Subretinal fluid rapidly resolved within $48 \mathrm{~h}$ and bestcorrected visual acuity improved to 0.5 LogMAR after 1 week. During the follow-up period of 12 months, no episodes of recurrence or reduction vision or adverse reactions were noted.

\section{Introduction}

Optic disc pit (ODP) is a rare congenital disease and known to involve temporal aspect of the optic nerve head, more commonly than the nasal side. It has an incidence of approximately one in 10,000 eyes [1]. ODP-associated maculopathy (ODP-M) characterized with accumulation of subretinal fluid occurs in $25-75 \%$ of patients with ODP [2], with 30 years as the mean age of presentation [3].

Many modalities have been described for the treatment of ODP-M. Few of which are vitrectomy with inner retinal fenestration, use of homologous scleral tissue, autologous 


\section{Case Reports in Ophthalmology}
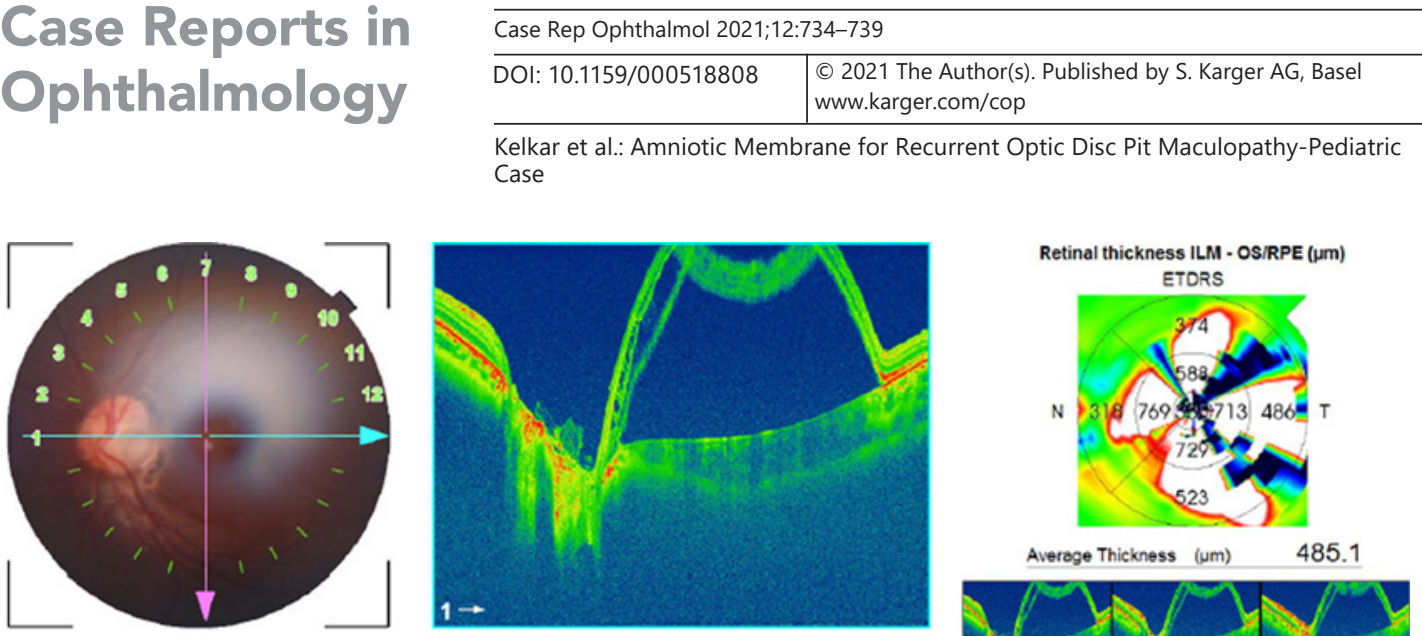

Kelkar et al.: Amniotic Membrane for Recurrent Optic Disc Pit Maculopathy-Pediatric Case
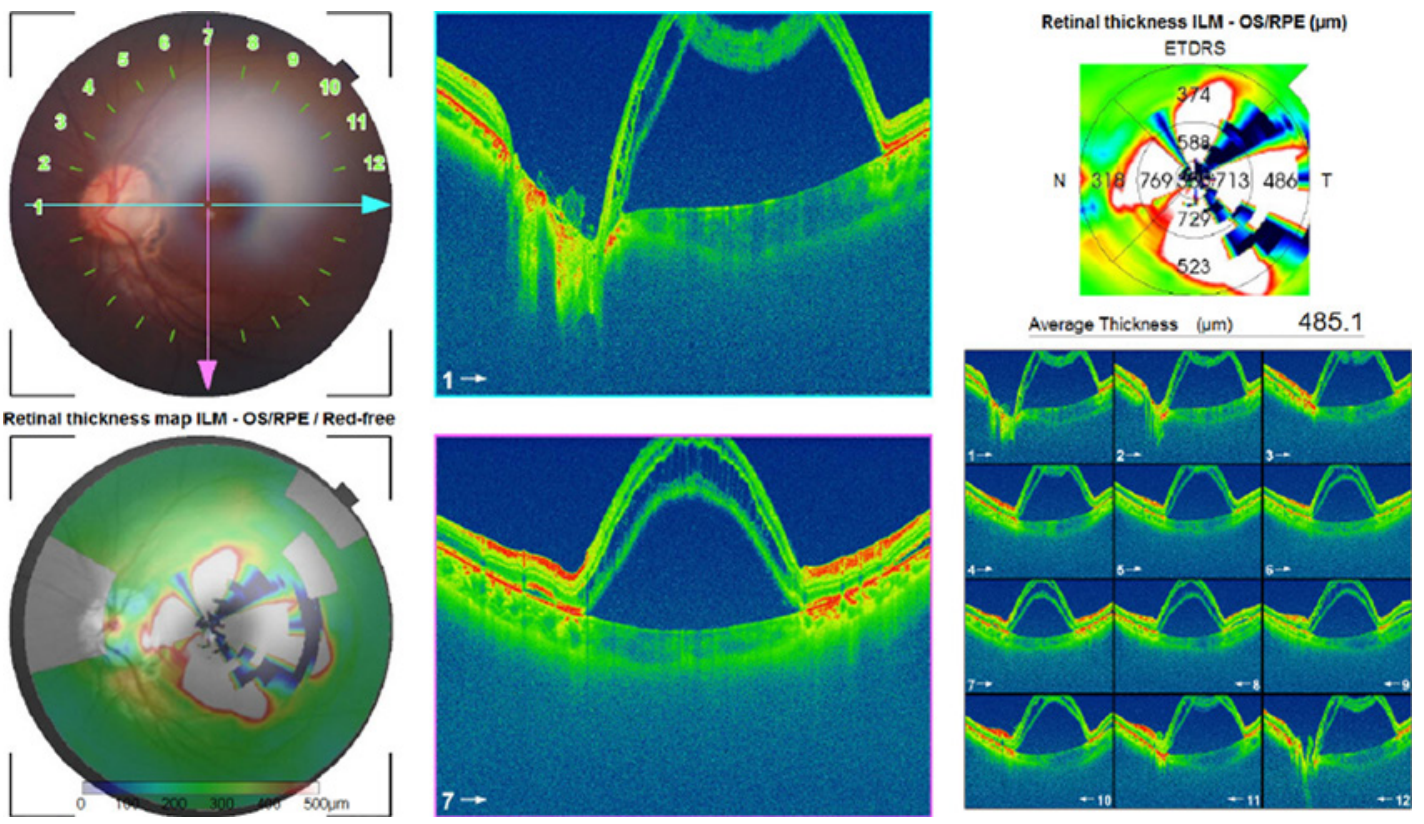

Fig. 1. Preoperative OCT showing with retinoschisis. ODP-M, optic disc pit-associated maculopathy; OCT, optical coherence tomography; ILM, internal limiting membrane.

platelet concentrate, fibrin glue, or internal limiting membrane (ILM) peel. However, there is no universally accepted treatment as pathophysiology of the disease has not yet been completely understood [4].

Rizzo et al. [5] recently used human amniotic membrane graft (hAMG) for the treatment of ODP-M in treatment naïve adult patients. We report a case of recurrent ODP-M in a pediatric case treated with hAMG that resulted in rapid resolution.

\section{Case Report}

A 13-year-old girl who was successfully operated 9 months ago for ODP-M with 25-G pars plana vitrectomy with double ILM flap technique, with one flap covering the ODP and the other one used to address the maculopathy along with SF6 gas tamponade and juxtapapillary endolaser, presented with 2-week history of painless, progressive diminution of vision in her left eye. Her best-corrected visual acuity in that eye was 2 LogMAR. She had no history of ocular trauma.

Fundus examination revealed a recurrence of ODP-M. With swept-source optical coherence tomography (OCT) (DRI Trition Plus; TOPCON, Itabashi City, Japan) a large localized bullous maculopathy with retinoschisis was noted (Fig. 1). Under general anesthesia, and under all aseptic precautions, 325 -gauge sclerotomy ports were made. A cryopreserved wet hAMG of size $3 \mathrm{~mm}$ was cut using a $3-\mathrm{mm}$ trephine, and the section was stained using brilliant blue stain $(0.05 \%)$. Thereafter, the stained hAMG was introduced into the eye with the help of intravitreal forceps and stuffed into the ODP. The proper position of the hAMG was confirmed by intraoperative OCT (Artevo, 800; Carl Ziess Meditech, Dublin, CA, USA) (Fig. 2) obviating the need for any additional, gas tamponade, laser, or perfluorocarbon liquid. Cannulas were gently removed and eye was patched. 


\section{Case Reports in Ophthalmology}

\begin{tabular}{l|l}
\hline Case Rep Ophthalmol 2021;12:734-739 \\
\hline DOI: 10.1159/000518808 & $\begin{array}{l}\text { @ } 2021 \text { The Author(s). Published by S. Karger AG, Basel } \\
\text { www.karger.com/cop }\end{array}$ \\
\hline
\end{tabular}

Kelkar et al.: Amniotic Membrane for Recurrent Optic Disc Pit Maculopathy-Pediatric Case

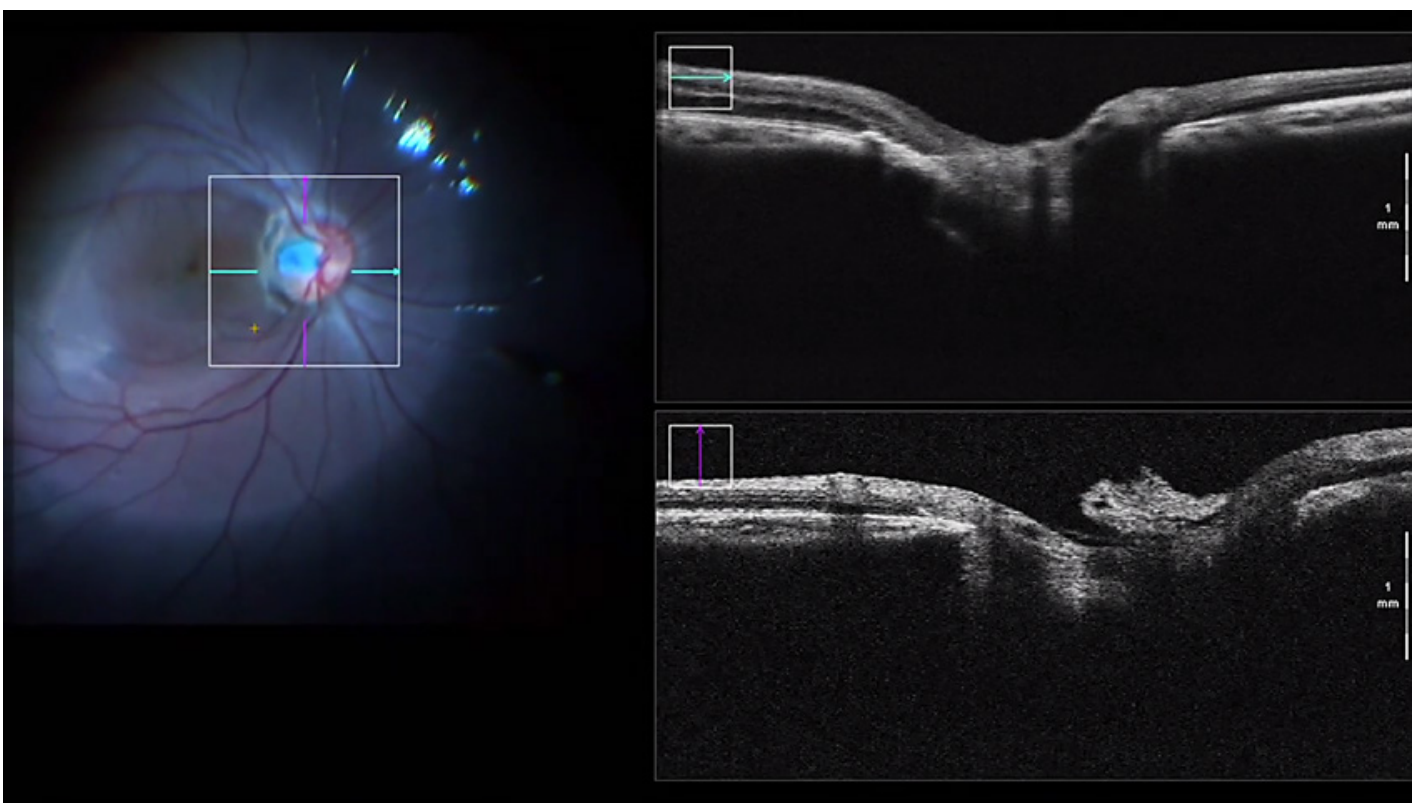

Fig. 2. Intraoperative OCT showing the snug fit of the hAMG. hAMG, Human Amniotic Membrane Graft; OCT, optical coherence tomography.

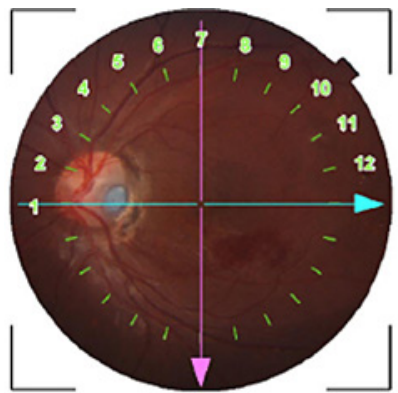

Retinal thickness map ILIM - OS/RPE / Red-free

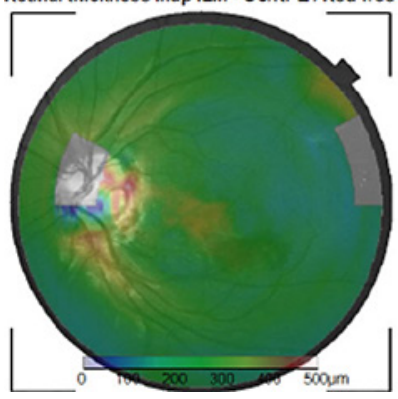

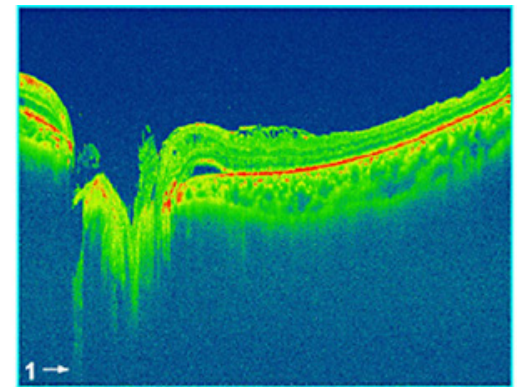

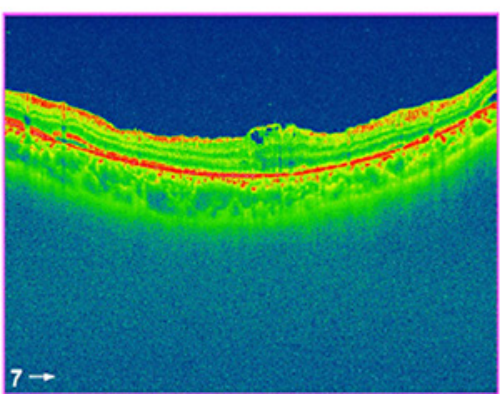

Retinal thickness ILM - OS/RPE (um) ETORS

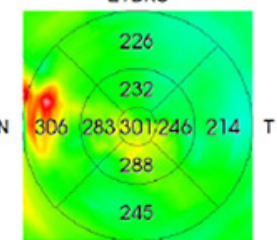

Average Thickness $(\mu \mathrm{m}) \quad 252.1$

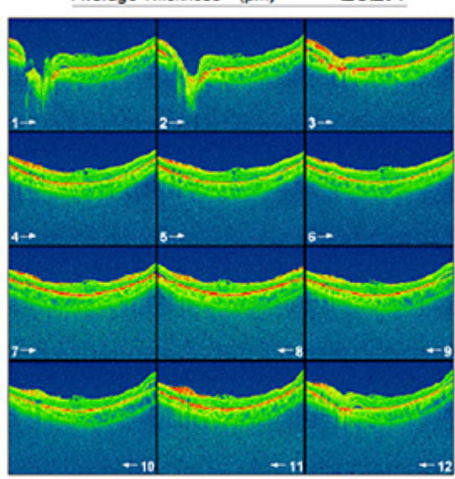

Fig. 3. Postoperative OCT showing resolution of the maculopathy within $48 \mathrm{~h}$ after the surgery. OCT, optical coherence tomography; ILM, internal limiting membrane.

We observed complete resolution of ODP-M within $48 \mathrm{~h}$ (Fig. 3) and best-corrected visual acuity improved to 0.5 LogMAR after 1 week. No complications or signs of recurrence were noted at 6 months or at 12-month follow-up, and the visual acuity was maintained at 0.5 LogMAR (Fig. 4, 5). 


\section{Case Reports in Ophthalmology}

\begin{tabular}{l|l}
\hline Case Rep Ophthalmol 2021;12:734-739 \\
\hline DOI: 10.1159/000518808 & $\begin{array}{l}\text { @ 2021 The Author(s). Published by S. Karger AG, Basel } \\
\text { www.karger.com/cop }\end{array}$ \\
\hline
\end{tabular}
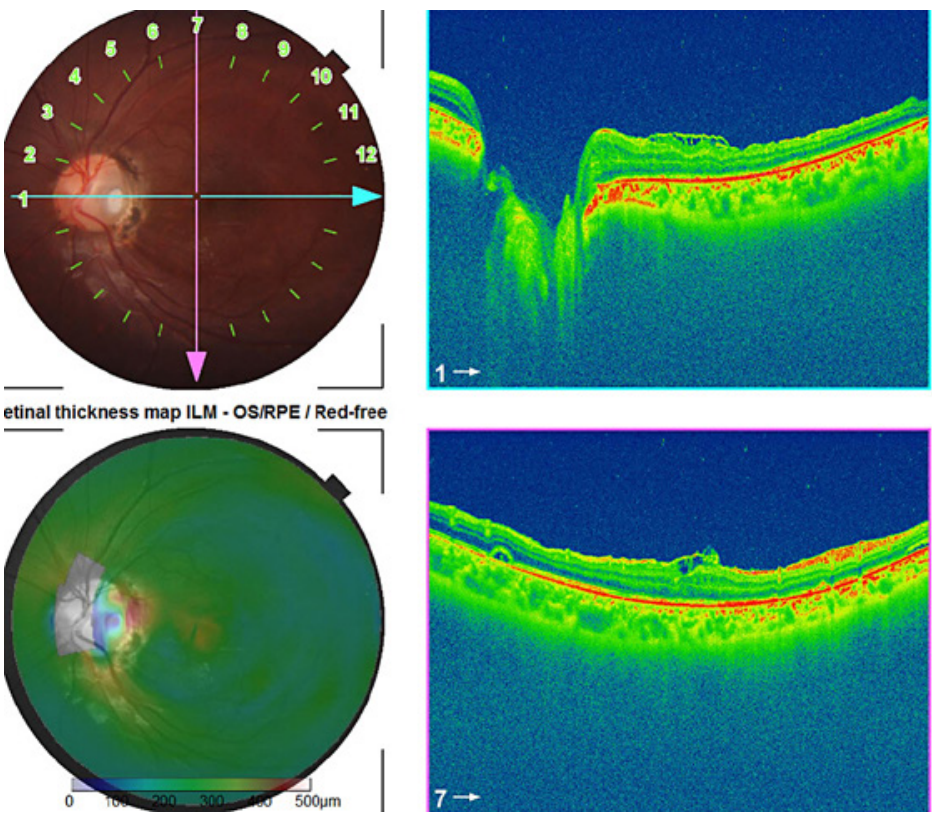

Kelkar et al.: Amniotic Membrane for Recurrent Optic Disc Pit Maculopathy-Pediatric Case
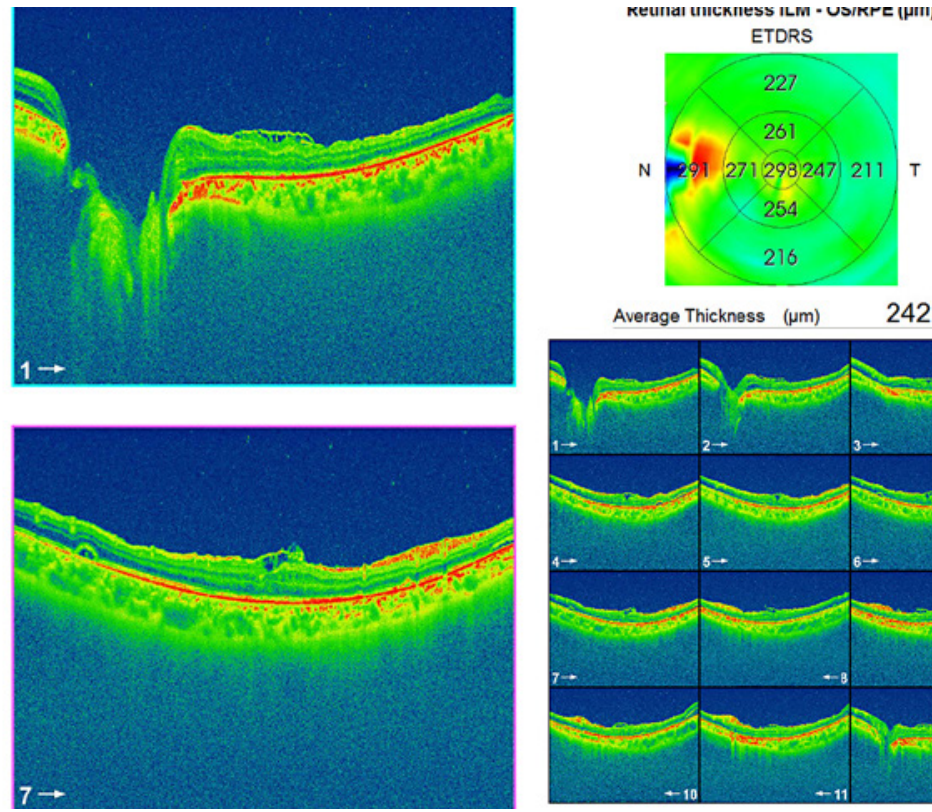

Average Thickness $(\mu \mathrm{m})$

242.5

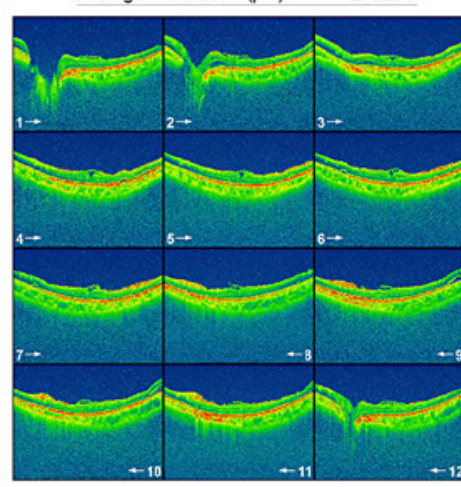

Fig. 4. Postoperative OCT showing no recurrence of ODP-M after 6 months. ODP-M, optic disc pit-associated maculopathy; OCT, optical coherence tomography; ILM, internal limiting membrane.

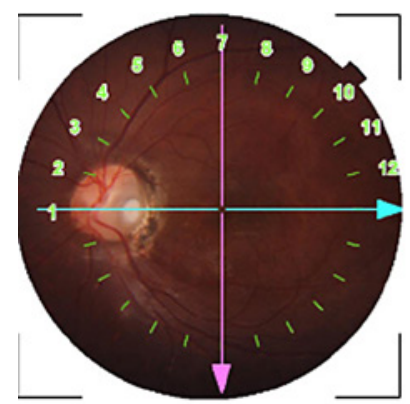

letinal thickness map ILM - OSIRPE / Red-free

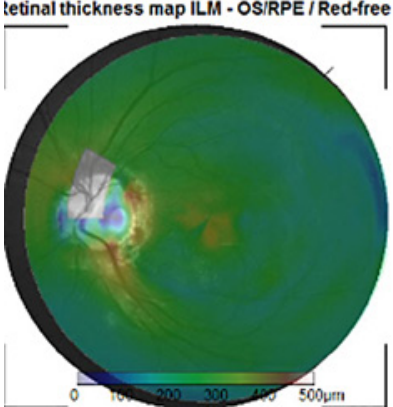

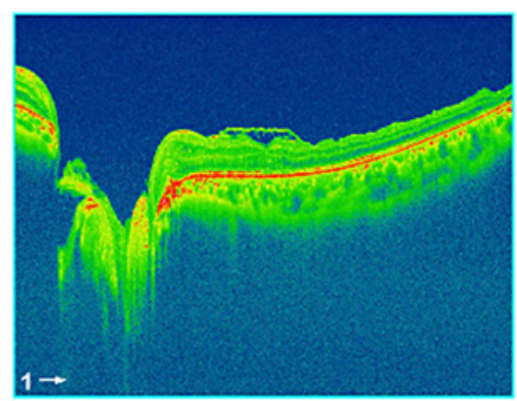

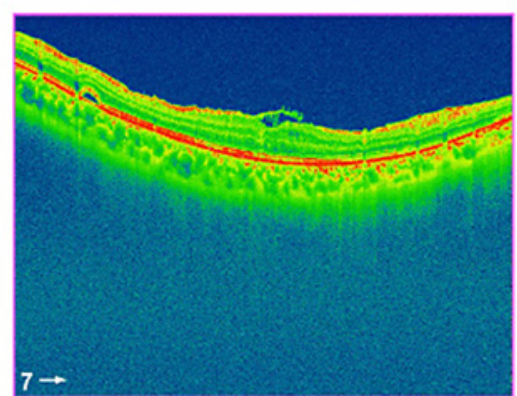

Retinal thickness ILM - OS/RPE $(\mu \mathrm{m})$ ETDRS

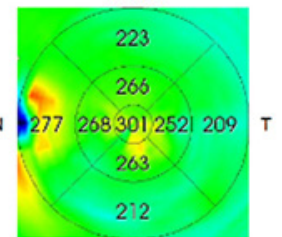

Average Thickness (um) 239.1

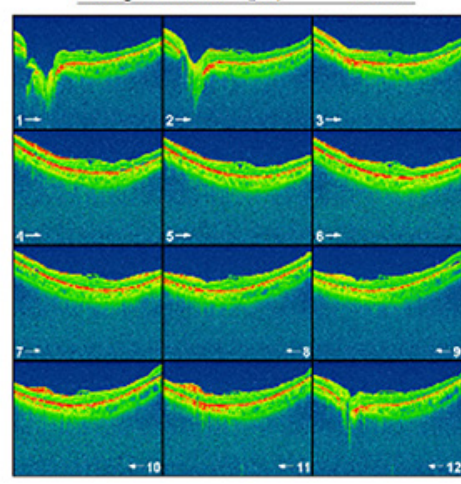

Fig. 5. Postoperative OCT showing no recurrence of ODP-M after 12 months. ODP-M, optic disc pit-associated maculopathy; OCT, optical coherence tomography; ILM, internal limiting membrane.

\section{Discussion}

ODP-M is a rare presentation in children [1]. Therefore, only a few pediatric cases successfully managed have been reported in the literature [3]. However, ODP-M in children usually presents with preceding incidents of trauma [6]. No such history of trauma was elicited in our 
case. The patient was operated previously by the same surgeon with double ILM flap with gas tamponade and juxtapapillary endolaser. Nine months following successful primary surgery, she presented to us with recurrence.

Use of hAMG in ophthalmology is not a new concept; it has been used since ages for anterior segment pathologies. Because of the malleable and pliable nature of the hAMG, it snugly fits in the optic nerve head pit better than rigid sclera, or fragile tissue such as ILM. Moreover, harvesting hAMG instead of harvesting a scleral flap drastically reduces the surgical time and also improves patient comfort.

Rizzo et al. [5] recently described an innovative surgical technique for management of ODP-M with 1.5-mm graft in treatment naïve adult patients. We used a larger 3-mm graft, due to the larger size of the ODP. This larger graft snuggly fit into the pit, as was confirmed by intraoperative OCT. Hence, there was no need of any additional adjuncts such as perfluorocarbon liquid or gas tamponade for stabilization of the graft.

Literature states that the average time taken for resolution of ODP-M ranges from 4 to 12 months [7]. We observed resolution within $48 \mathrm{~h}$. This may be attributed to the snug fit of the larger graft and to the already vitrectomized status of the eye.

\section{Conclusion}

Use of hAMG for ODP-M is new and to the best of our knowledge, this is the first case report of recurrent ODP-M treated with hAMG in a pediatric case.

\section{Statement of Ethics}

This study followed the tenets of the Declaration of Helsinski. This study protocol was reviewed and the need for approval was waived by NIO Ethics Committee, affiliated to National Institute of Ophthalmology, Pune. Written informed consent was obtained from the patient's parents for the publication of this case report, including medical record details, fundus photos, OCT images, and ancillary testing.

\section{Conflict of Interest Statement}

The author(s) declared no potential conflicts of interest with respect to the research, authorship, and/or publication of this article.

\section{Funding Sources}

The author(s) received no financial support for the research, authorship, and/or publication of this article.

\section{Author Contributions}

All the authors have contributed significantly in this case report. 


\section{Data Availability Statement}

All data generated or analyzed during this study are included in this article. Further enquiries can be directed to the corresponding author.

\section{References}

1 Greear JN. Pits, or crater-like holes, in the optic disk. Arch Ophthalmol. 1942 Sep 1;28(3):467-83.

2 Brown GC, Augsburger JJ. Congenital pits of the optic nerve head and retinochoroidal colobomas. Can J Ophthalmol. 1980 Jul;15(3):144-6.

3 Georgalas I, Petrou P, Koutsandrea C, Papaconstadinou D, Ladas I, Gotzaridis E. Optic disc pit maculopathy treated with vitrectomy, internal limiting membrane peeling, and gas tamponade: a report of two cases. Eur J Ophthalmol. 2009;19:324-6.

4 Uzel MM, Karacorlu M. Optic disk pits and optic disk pit maculopathy: a review. Surv Ophthalmol. 2019 Sep 1; 64(5):595-607.

5 Rizzo S, Caporossi T, Pacini B, De Angelis L, De Vitto ML, Gainsanti F. Management of optic disk pit-associated macular detachment with human amniotic membrane patch. Retina. 2020 Jan 10. Epub ahead of print.

6 Rii T, Hirakata A, Inoue M. Comparative findings in childhood-onset versus adult-onset optic disc pit maculopathy. Acta Ophthalmol. 2013 Aug;91(5):429-33.

7 Zheng A, Singh RP, Lavine JA. Surgical options and outcomes in the treatment of optic pit maculopathy: a metaanalysis and systematic review. Ophthalmol Retina. 2020 Mar 1;4(3):289-99. 